\title{
Qualidade da matéria orgânica e distribuição do fósforo no solo de lavouras orgânicas de café Conilon
}

\author{
Organic matter quality and phosphorus distribution in soils under organic Conilon coffee
}

\author{
Fábio Luiz Partelli ${ }^{I}$ Jader Galba Busato ${ }^{I I}$ Henrique Duarte VieiraII Alexandre Pio Viana ${ }^{\text {II }}$ \\ Luciano Pasqualoto Canellas ${ }^{\text {II }}$
}

\section{RESUMO}

O cultivo de café orgânico utiliza fontes não solúveis de fósforo e grande quantidade e variedade de material orgânico em seu manejo. Assim, o objetivo do trabalho foi avaliar as frações que compõem a matéria orgânica $e$ distribuição do fósforo no solo de cafeeiros Coffea canephora da cultivar 'Conilon' cultivados sob diferentes sistemas de manejos de produção orgânica. $O$ solo foi coletado na projeção da copa do cafeeiro, na camada de 0 a $20 \mathrm{~cm}$. Determinaramse as características químicas e granulométricas do solo em procedimentos de rotina e as frações de fósforo e da material orgânica. Na maioria das lavouras, o fósforo orgânico constituiu a maior parte do fósforo lábil, e a fração humina constituiu a maior parte da matéria orgânica. Houve maior presença de fósforo inorgânico nos solos das lavouras com maiores concentrações de fósforo total e lábil. O conteúdo total de $\mathrm{Pi}+\mathrm{Po}$ nos solos avaliados apresentou valores elevados, variando de 426,9 até $910,4 \mathrm{mg} \mathrm{dm}^{-3}$ de solo. A fração humina constituiu a maior parte da matéria orgânica. As frações que compõem a distribuição do fósforo no solo apresentaram discrepâncias entre os sistemas de manejos de produção orgânica.

Palavras-chave: Coffea canephora, agricultura orgânica, substâncias húmicas, fósforo.

\section{ABSTRACT}

The organic management in coffee uses insoluble phosphorus sources and a large quantity and variety of organic material. Thus, the objective of this research was to evaluate organic matter composition and phosphorus distribution on soils from Coffea canephora cv. 'Conilon' crops, under different organic management production systems. Soil under the coffee tree canopy was collected from 0 to $20 \mathrm{~cm}$ depth. Chemical and soil texture characteristics were determined by routine analysis as well as phosphorus and organic matter constitution. On the average crops organic phosphorus was the main part of the labile phosphorus and carbon humine. There was a higher content of inorganic phosphorus on soils from the crops with the highest concentrations of total and labile phosphorus. The total content of $\mathrm{Pi}+\mathrm{Po}$ in the evaluated soils showed high and variation from 426.9 to $910.4 \mathrm{mg} \mathrm{dm}^{-3}$ of soil. The humin fraction formed the bulk of organic matter. The fractions that make up the phosphorus distribution in the soil showed discrepanncies between the organic management production systems.

Key words: Coffea canephora, organic agriculture, humic substances, phosphorus.

\section{INTRODUÇÃO}

Culturas exploradas de forma inadequada podem acarretar degradação do solo, perda de fertilidade, aumento do ataque de pragas e doenças e degradação do ambiente (PRIMAVESI, 1992). Nesse contexto, a agricultura orgânica desponta como uma solução para mitigar parte desses problemas, podendo promover um desenvolvimento rural mais sustentável (THEODORO et al., 2003). Na lavoura orgânica de café, não é realizada a adubação com fertilizantes solúveis e nem o uso de agrotóxicos, o que é substituído pela adubação com resíduos orgânicos compostados ou não, fosfatos naturais e cobertura verde e pelo controle fitossanitário e de plantas daninhas sem o uso de agrotóxicos. Isso provoca alterações das frações que compõem a matéria orgânica, na disponibilidade de fósforo e demais nutrientes, além da atividade microbiana do

\footnotetext{
IUniversidade Federal de Goiás (UFG), Escola de Agronomia e Engenharia de Alimentos (EA), Campus Samambaia, CP 131, 74001970, Goiânia, GO, Brasil. E-mail: partelli@yahoo.com.br. Autor para correspondência.

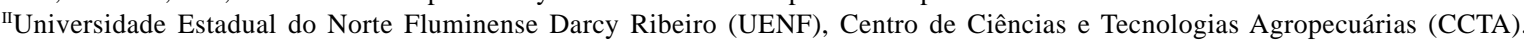
Campo dos Goytacazes, RJ, Brasil.
} 
solo (MALUCHE-BARETTA et al., 2006). Sistemas de cultivo orgânico, em particular de café Conilon cultivados no Espírito Santo, apresentam limitações de P no solo e nas folhas, sendo, geralmente, suprida pela aplicação de fosfatos naturais (PARTELLI et al., 2006).

Nos ambientes tropicais, a maior parte da matéria orgânica do solo é formada pelas substâncias húmicas (SH), produto da transformação biológica dos resíduos orgânicos até formas estabilizadas de compostos relativamente refratários à decomposição biológica. De acordo com a sua solubilidade, as SH podem ser classificadas em ácidos fúlvicos (AF), ácidos húmicos (AH) e huminas (H) (ZECH et al., 1997). A distribuição relativa das frações humificadas podem ser utilizadas como indicador da qualidade da matéria orgânica (MACCALLISTER \& CHIEN, 2000).

O manejo do solo pode modificar a dinâmica do fósforo, alterando o conteúdo das diferentes formas de $\mathrm{P}$. O fracionamento do $\mathrm{P}$ permite constatar as diferentes distribuições deste em diversas classes de solo (GUERRA et al., 1996), bem como o efeito do tempo de cultivo sob diferentes formas de manejo, numa mesma classe de solo, caracterizando suas formas presentes no solo (TOKURA et al., 2002). As análises de rotina para a avaliação da fertilidade do solo abrangem somente o P inorgânico e a matéria orgânica, havendo poucos estudos direcionados para a avaliação dos compartimentos orgânicos e do fracionamento do P, principalmente, em lavouras sob sistema orgânico de produção.

Assim, o objetivo deste trabalho foi avaliar as frações que compõem a matéria orgânica e a distribuição do fósforo no solo de cafeeiros Conilon cultivados sob diferentes manejos de produção orgânica.

\section{MATERIAL E MÉTODOS}

Foram avaliadas oito lavouras de café Conilon (C. canephora). As lavouras eram cultivadas no sistema orgânico (BRASIL, 2003), certificadas ou em processo de certificação, sem o uso de agrotóxicos e com utilização de resíduos orgânicos e rochas fosfatadas (Tabela 1). As lavouras estão localizadas no Norte do Espírito Santo, numa região caracterizada pelo clima tropical, quente e úmido no verão e inverno seco, com precipitação anual em torno de $1200 \mathrm{~mm}$ e temperatura média $23^{\circ} \mathrm{C}$, com a maioria dos solos classificados como Latossolo Vermelho-Amarelo (ANA, 2004).

Para caracterização química do solo das oito lavouras, foram obtidas três amostras compostas provenientes de 15 subamostras simples coletadas numa profundidade de $0-0,2 \mathrm{~m}$, na projeção da copa do cafeeiro. Para a análise foliar, as folhas do cafeeiro foram coletadas, aleatoriamente, no terceiro e/ou no quarto nó dos ramos plagiotrópicos localizado no terço mediano superior da planta. As análises foliares de fósforo e de solo (P, K, Ca, Mg e granulométricas) foram realizadas conforme SILVA(1999).

O fracionamento da matéria orgânica foi realizado em triplicata, utilizando $\mathrm{NaOH} 0,5 \mathrm{~mol} \mathrm{~L}^{-1}$ como extrator na relação solo: solvente de 1:20 (m/v). Efetuouse a agitação do material durante $6 \mathrm{~h}$ com posterior centrifugação (3500g durante 30 minutos). Esse procedimento foi repetido até o sobrenadante apresentar-se incolor. O extrato solúvel foi reunido e teve o $\mathrm{pH}$ ajustado até 1-1,5, utilizando-se $\mathrm{HCl} 6 \mathrm{~mol}$ $\mathrm{L}^{-1}$. Os ácidos húmicos foram separados dos ácidos fúlvicos por centrifugação. O resíduo sólido resultante da extração com $\mathrm{NaOH}$, denominado fração humina, foi recolhido e seco em estufa $\left(50^{\circ} \mathrm{C}\right)$. O conteúdo de $\mathrm{C}$ em cada fração foi determinado por meio de dicromatometria, utilizando a metodologia usada e indicada pela Sociedade Internacional de Substâncias húmicas (SWIFT, 1996).

Para a quantificação do $\mathrm{P}$ nas formas orgânicas (Po) e inorgânicas (Pi), utilizou-se a metodologia proposta por BOWMAN (1989), seguindo as modificações sugeridas por GUERRA et al. (1996), realizadas em triplicata. Em amostras de solo, foram adicionados $\mathrm{H}_{2} \mathrm{SO}_{4}$ concentrado e água. Em seguida, o material foi agitado e centrifugado, e o sobrenadante foi filtrado em papel quantitativo faixa azul e, logo após, foi determinado o $\mathrm{P}$ total ácido. No restante do extrato, foi adicionado carvão ativo lavado com $\mathrm{HCl}\left(6 \mathrm{~mol} \mathrm{~L}^{-1}\right)$, para determinação do Pi ácido. Ao solo remanescente no tubo de centrifuga foi adicionado $\mathrm{NaOH}\left(0,5 \mathrm{~mol} \mathrm{~L}^{-1}\right)$. O material foi submetido a banho-maria e, após o resfriamento, os tubos de centrífuga foram novamente agitados, centrifugados e filtrados. Em seguida, foi determinado o $\mathrm{P}$ total alcalino. Ao restante do extrato, adicionou-se carvão ativo para determinação do Pi alcalino. O conteúdo de $\mathrm{P}$ orgânico total foi estimado a partir da diferença entre o $\mathrm{P}$ total dos estratos ácido e alcalino e seus respectivos valores de Pi.

A quantificação do P lábil foi realizada de acordo com BOWMAN \& COLE (1978). Para tal foi utilizado bicarbonato de sódio, e as amostras passaram por agitação, decantação, utilização de carvão ativo purificado e digestão com ácido perclórico concentrado. A quantificação do $\mathrm{P}$ nos extratos foi realizada por meio de espectrofotômetria no espectro visível, a partir da formação da cor azul, complexo fosfato-molibdato em solução ácida, utilizando-se o ácido ascórbico (MURPHY \& RILEY, 1962).

Foram realizados estudo descritivo dos dados, análise multivariada (componentes principais) para definir grupos de lavouras e estimadas as correlações simples por meio do método de simulação de subamostras com diversas combinações de números de amostras, ficando de fora das estimativas as correlações acima ou abaixo do intervalo 0 - 1, método denominado de Bootstrap (CRUZ, 2001). 
Tabela 1 - Identificação e algumas características e manejos adotados no último ano, nas oito lavouras de café Conilon da região Norte do Espírito San to cultivad as sob sistema orgâni co desde o plantio.

\begin{tabular}{|c|c|c|c|c|}
\hline Lavoura & $\begin{array}{l}\text { Idade } \\
\text { (anos) }\end{array}$ & $\begin{array}{l}\text { Espaçamento } \\
\text { (m) }\end{array}$ & sac as ha-1/ Muni cipi o & Manejo adotado no último ano \\
\hline 1 & 3 & $2 \times 1$ & 22 / Boa Esperança & $\begin{array}{l}\text { Irrigada por gotejamento, } 1 \mathrm{Mg} \text { ha }^{-1} \text { de calcário, } 100 \mathrm{~g} \text { planta }^{-1} \text { de } \\
\text { fosfato natural, } 5 \text { litros planta }{ }^{-1} \text { de composto orgânico, pulveri zação de } \\
\text { super magro }{ }^{1} \text { ( } 6 \text { vezes ao ano), urina de vaca }{ }^{2} \text {, capina com enxad a e } \\
\text { consórcio tend o Ricinus com munis e Canavalia ensiformis. }\end{array}$ \\
\hline 2 & 6,5 & $2,5 \times 2$ & 32 / São Mateus & $\begin{array}{l}\text { Irrigada por gotejamento, } 1 \mathrm{Mg} \mathrm{ha}^{-1} \text { de calcário, } 1 \mathrm{Mg} \mathrm{ha}^{-1} \text { de } \mathrm{MB}^{3} \text {, } \\
9 \mathrm{~kg}^{-1} \text { ano }^{-1} \text { planta }{ }^{-1} \text { de húmus de minhoca, capina com enxada e foice e } \\
\text { consórcio utilizando Cajanus cajan e Macadamia integrifolia. }\end{array}$ \\
\hline 3 & 6 & $2,5 \times 1$ & 20 / J aguaré & $\begin{array}{l}\text { Irrigada por gotejamento, } 1 \mathrm{Mg} \mathrm{ha}^{-1} \text { de calcário, } 50 \mathrm{~g} \mathrm{plant}^{-1} \text { de fosfato } \\
\text { natural, } 0,8 \mathrm{Mg} \mathrm{ha}^{-1} \text { de } \mathrm{MB}^{3}, \mathrm{Microgel}^{4} \text {, } 5 \text { litros planta }{ }^{-1} \text { de composto } \\
\text { orgânico, urina de vaca }{ }^{2} \text {, capina utilizando enxada e foice e consórcio } \\
\text { com C. cajan. }\end{array}$ \\
\hline 4 & 6 & $2,5 \times 1$ & 20 / J aguaré & $\begin{array}{l}\text { Irrigada por gotejamento, } 1 \mathrm{Mg} \mathrm{ha}^{-1} \text { de cal cánio, } 0,8 \mathrm{Mg} \mathrm{ha}^{-1} \text { de } \mathrm{MB}^{3} \text {, } \\
\text { Microgel }^{4}, 10 \text { litros planta }^{-1} \text { de composto, urina de } \mathrm{vaca}^{2} \text {, capin a com } \\
\text { enxada e foi ce e consórcio com } \text { C. cajan. }\end{array}$ \\
\hline 5 & 3,5 & $2,3 \times 1,1$ & 26 / J aguaré & $\begin{array}{l}\text { Irrigada por gotej amento, } 1,3 \mathrm{Mg} \mathrm{ha}^{-1} \text { de calcário, } 1,5 \mathrm{Mg} \mathrm{ha}^{-1} \text { de } \mathrm{MB}^{3} \text {, } \\
\text { Microgel } \mathrm{l}^{4} \text {, } 3 \text { litros planta }{ }^{-1} \text { de composto orgânico, capina utilizando } \\
\text { enxada e foi ce e consórcio com } \text { C. cajan. }\end{array}$ \\
\hline 6 & 7 & $1,7 \times 1,5$ & 24 / Jaguaré & $\begin{array}{l}\text { Não foi irrigada, } 0,6 \mathrm{Mg} \text { ha }^{-1} \text { de cal cário, } 0,6 \mathrm{Mg} \mathrm{ha}^{-1} \text { de } \mathrm{MB}^{3} \text {, } \\
\text { Microgel } \mathrm{l}^{4} \text {, } 8 \text { litros planta }{ }^{-1} \text { de compost o orgânico, capina seletiva e } \\
\text { foice e consórcio com Pueraria phaseoloides. }\end{array}$ \\
\hline 7 & 6 & $2,5 \times 1,7$ & 24 / Rio Bananal & $\begin{array}{l}\text { Irrigada por aspersão convencional, capina utilizando enxada e foice e } \\
\text { consórcio com } \boldsymbol{M} \text { usa spp. }\end{array}$ \\
\hline 8 & 5 & $2,5 \times 1$ & $\begin{array}{l}85 \text { / São Domingos } \\
\text { do Norte }\end{array}$ & $\begin{array}{l}\text { Irrigada por aspers ão convencional, } 2,5 \mathrm{Mg} \mathrm{ha}^{-1} \text { de cal cário, } 0,8 \mathrm{Mg} \mathrm{ha}^{-1} \\
\text { de } \mathrm{MB}^{3}, 10 \text { litros planta }{ }^{-1} \text { de co mposto orgânico, } 10 \text { litros planta }{ }^{-1} \text { de } \\
\text { palha de café, urina de vaca }{ }^{2} \text {, capina utilizando enxada e foice e } \\
\text { consórcio com } C \text {. en siformis. }\end{array}$ \\
\hline
\end{tabular}

${ }^{1}$ Biofertilizante produzido com sulfato de $\mathrm{Zn}, \mathrm{Cu}, \mathrm{Mn}, \mathrm{Mg}, \mathrm{Fe}, \mathrm{CaCl}_{2}$, ácido bórico, molibdato de amôn io, Fosfato de Araxá, calcário, soro de leite e melaço de cana, ${ }^{2} 1$ a 2 litro de urina de vaca para 20 litros de água, ${ }^{3} 479,6 \mathrm{SiO}_{2}, 22,3 \mathrm{CaO}, 191,4 \mathrm{MgO}_{(\mathrm{g} \mathrm{Kg}}{ }^{-1}$ ) e ${ }^{4} \mathrm{Produto}$ comercial com finalidade de melhorar a decomposição do esterco.

\section{RESULTADOS E DISCUSSÃO}

Ao realizar a análise multivariada (componentes principais), verificou-se que o Po pouco lábil, o P extraído pelo Mehlich e o Pi associado aos óxidos apresentaram os maiores autovalores, os quais, junto com outras características avaliadas, permitiram agrupar as lavouras orgânicas em quatro grupos: grupo 1 (formado pelas lavouras 1,3 e 4), grupo 2 (lavouras 2 , 5 e 6 ) e os grupos 3 e 4 formados pelas lavouras 7 e 8 , respectivamente (Figura 1). Ao relacionar esses grupos com o manejo (Tabela 1), observa-se que duas lavouras do grupo 1 (lavouras 1 e 3) receberam aplicação de fosfato natural e apresentam, nas três lavouras, maior concentração foliar de $\mathrm{P}$, saturação de bases superior a 70\%, maiores valores de $\mathrm{pH}$, soma de bases trocáveis e P pelo método de Mehlich (Tabela 2), bem como maiores valores de Pi lábil e não lábil e Pi + Po total e lábil (Tabela 3 ) em relação aos demais grupos. O grupo 2 diferencia-se das lavouras 7 e 8 por apresentar menores porcentagens de argila e menor capacidade de troca catiônica.
O conteúdo de $\mathrm{P}$ total nos solos avaliados apresentou valor médio de $851,92 \mathrm{mg} \mathrm{kg}^{-1}$ de solo, variando de 655 até $976 \mathrm{mg} \mathrm{dm}^{-3}$ solo (Tabela 2), sendo compatível com a maioria dos solos brasileiros analisados por GUERRA et al. (1996), ALMEIDA et al. (2003) e NUÑES et al. (2003). Apesar de serem observados valores de $\mathrm{P}$ total relativamente elevados nas lavouras 2, 5, 6, 7 e 8, o conteúdo de P disponível nesses solos foi baixo (Tabela 2 e 3), o que pode estar relacionado com a elevada capacidade de retenção de fósforo em solos altamente intemperizados (NOVAIS \& SMITH, 1999).

O conteúdo de Po total variou de 199,9mg $\mathrm{dm}^{-3}$ de solo na lavoura 8 até $310,5 \mathrm{mg} \mathrm{kg}^{-1}$ de solo na lavoura 3 (Tabela 3), com média de $245,9 \mathrm{mg} \mathrm{dm}^{-3} \mathrm{de}$ solo. No presente trabalho, o Po total representou de 29 a 56,9\% do P total e, nas lavouras 2, 5 e 6, essa fração foi superior ao Pi total. A participação do Pi representou 43 a $71 \%$ do $\mathrm{P}$ total, sendo mais expressivos nas lavouras 1, 3 e 4 (Tabela 3). Embora em termos médios a participação do Pi seja superior à do Po (GUERRA et al., 1996; CUNHA et al., 2003; NUÑES et al., 2003), o valor médio de $43 \%$ de Po é expressivo, 


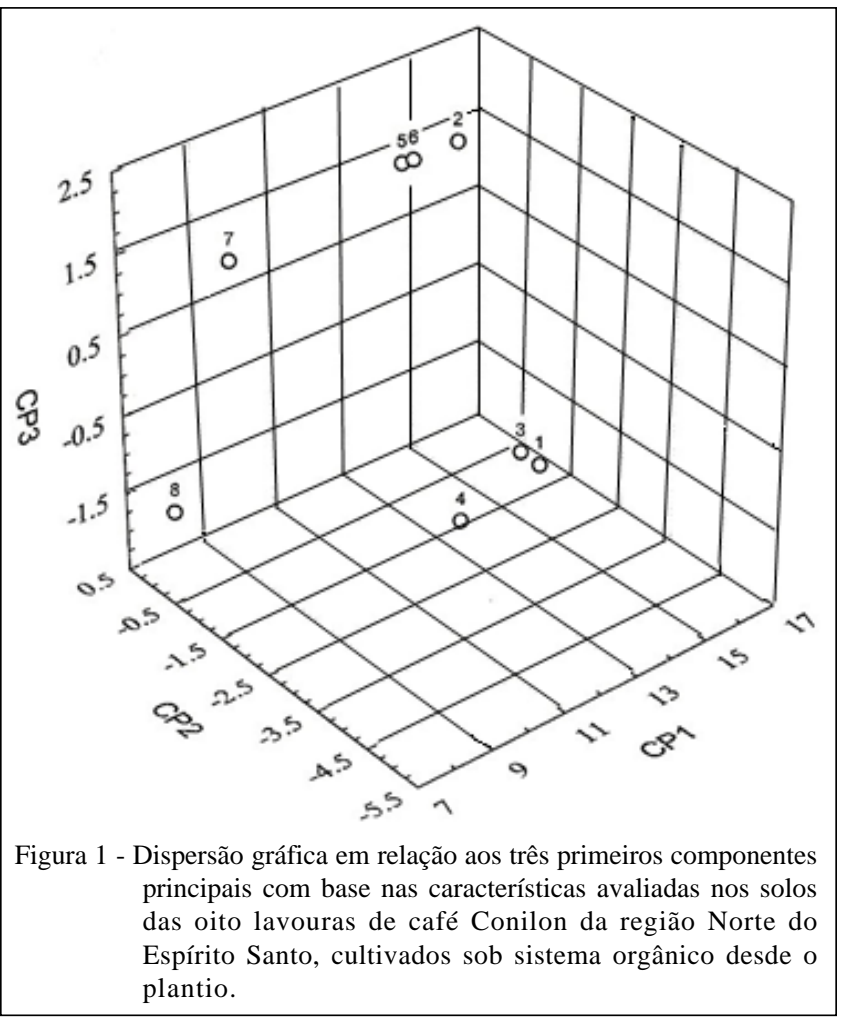

uma vez que no trabalho de GUERRA et al. (1996) esse valor é menor para a grande maioria dos solos estudados, podendo também ser um indicativo da mudança de comportamento no ciclo do $\mathrm{P}$ em função do manejo orgânico adotado. Assim, os resultados observados, comparados aos dados apresentados por GUERRA et al. (1996), permitem inferir que as atividades adotadas no sistema de produção orgânica, como o menor revolvimento do solo e as plantas de cobertura, a permanência de resíduos vegetais e a adição de materiais orgânicos podem favorecer a manutenção e o acúmulo do fósforo orgânico e lábil, corroborando MATOS et al. (2006), os quais descrevem que os aumentos nos teores das formas mais lábeis de $\mathrm{P}$, proporcionados pela adubação orgânica, evidenciam a importância desse sistema de manejo no favorecimento da ciclagem de $P$.

Embora o Pi seja predominante em relação ao $\mathrm{P}$ total, a maior parte do $\mathrm{P}$ lábil é constituída pelo Po (Tabela 3). A utilização de vegetação nas entrelinhas da lavoura e o uso de resíduos orgânicos podem ter favorecido o acúmulo de P orgânico e lábil no solo, como observado por NUÑES et al. (2003) em sistema de cultivo mínimo e com utilização de vegetação de cobertura. GATIBONI et al. (2007) descrevem que, em solos com baixa ou nenhuma adição de adubos fosfatados, as formas orgânicas de P são as principais mantedoras do $\mathrm{P}$ absorvido pelas plantas,

Tabela 2 - Concentração foliar de fósforo, granulometria e características químicas no solo das oito lavouras de café Conilon da região Norte do Espírito Santo, cultivados sob sistema orgânico desde o plantio

\begin{tabular}{|c|c|c|c|c|c|c|c|c|c|c|c|c|c|c|c|c|c|}
\hline Lavoura & $\mathrm{pH}^{1}$ & $\mathrm{P}^{2}$ & $\mathrm{P}^{3}$ & $\mathrm{P}^{4}$ & $\mathrm{P}^{5}$ & $\mathrm{~K}^{6}$ & $\mathrm{Ca}^{7}$ & $\mathrm{Mg}^{7}$ & $\mathrm{Al}^{6}$ & $\mathrm{H}+\mathrm{Al}^{7}$ & SB & $\mathrm{t}$ & CTC & V & Areia & Silte & Argila \\
\hline- & - & \multicolumn{2}{|c|}{$\mathrm{mg} \mathrm{kg}^{-1}$} & \multicolumn{3}{|c|}{----mg dm-3---- } & \multicolumn{7}{|c|}{ 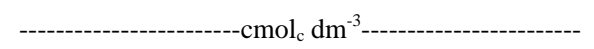 } & \multicolumn{4}{|c|}{-------------dag kg-1------------- } \\
\hline 1 & 6,1 & 0,20 & 655 & 35,0 & 15 & 163 & 2,37 & 0,7 & 0 & 1,34 & 3,51 & 3,51 & 4,86 & 72,4 & 79 & 1 & 20 \\
\hline EPM & 0,1 & - & 43 & 1,3 & 0,9 & 0,9 & 0,01 & 0,01 & 0 & 0,09 & 0,05 & 0,05 & 0,03 & 1,59 & - & - & - \\
\hline 2 & 5,6 & 0,18 & 717 & 5,8 & 50 & 66 & 1,61 & 0,85 & 0 & 1,99 & 2,64 & 2,64 & 4,64 & 57,4 & 75 & 2 & 23 \\
\hline EPM & 0 & - & 51 & 0,5 & 1,7 & 1,4 & 0,02 & 0,01 & 0 & 0,19 & 0,04 & 0,06 & 0,14 & 2,80 & - & - & - \\
\hline 3 & 6,3 & 0,21 & 924 & 86,2 & 26 & 132 & 3,37 & 1,14 & 0 & 1,28 & 4,9 & 4,9 & 6,15 & 79,3 & 77 & 2 & 21 \\
\hline EPM & 0,1 & - & 76 & 2,7 & 0,9 & 2,3 & 0,03 & 0,00 & 0 & 0,12 & 0,05 & 0,05 & 0,09 & 1,73 & - & - & - \\
\hline 4 & 6,0 & 0,19 & 912 & 63,4 & 32 & 124 & 2,66 & 1,34 & 0 & 1,60 & 4,38 & 4,38 & 5,96 & 73,4 & 64 & 6 & 30 \\
\hline EPM & 0 & - & 84 & 2,0 & 1,2 & 1,2 & 0,05 & 0,02 & 0 & 0,15 & 0,07 & 0,07 & 0,10 & 2,11 & - & - & - \\
\hline 5 & 5,8 & 0,12 & 872 & 7,2 & 4 & 80 & 1,76 & 0,48 & 0 & 1,68 & 2,46 & 2,46 & 4,14 & 59,7 & 83 & 3 & 14 \\
\hline EPM & 0 & - & 73 & 0,4 & 0,0 & 0,9 & 0,03 & 0,02 & 0 & 0,11 & 0,03 & 0,64 & 0,14 & 1,27 & - & - & - \\
\hline 6 & 4,9 & 0,16 & 856 & 4,0 & 4 & 153 & 1,55 & 0,59 & 0,1 & 2,66 & 2,54 & 2,64 & 5,21 & 49,6 & 57 & 9 & 34 \\
\hline EPM & 0 & - & 70 & 0,4 & 0,3 & 2,0 & 0,03 & 0,01 & 0 & 0,34 & 0,04 & 0,06 & 0,29 & 3,61 & - & - & - \\
\hline 7 & 4,6 & 0,12 & 877 & 4,9 & 6 & 75 & 2,22 & 0,53 & 0,5 & 6,2 & 2,97 & 3,47 & 9,15 & 33,6 & 36 & 12 & 52 \\
\hline EPM & 0 & - & 69 & 0,0 & 0,3 & 1,2 & 0,01 & 0,01 & 0,01 & 0,97 & 0,02 & 0,00 & 0,96 & 3,67 & - & - & - \\
\hline 8 & 4,9 & 0,16 & 976 & 3,9 & 5 & 108 & 1,68 & 0,76 & 0,4 & 4,34 & 2,75 & 3,15 & 7,09 & 39,4 & 42 & 9 & 49 \\
\hline EPM & 0 & - & 98 & 0,2 & 0,6 & 1,2 & 0,01 & 0,01 & 0,01 & 0,41 & 0,14 & 0,14 & 0,28 & 3,44 & - & - & - \\
\hline CV & 1,5 & - & 8,7 & 8,7 & 8,9 & 2,2 & 3,5 & 2,8 & 4,8 & 6,7 & 3,4 & 11,6 & 11,1 & 8,0 & - & - & - \\
\hline
\end{tabular}

${ }^{1}$ Em água, ${ }^{2}$ Fósforo foliar, ${ }^{3}$ Fósforo por digestão sulfúrica, ${ }^{4}$ Pósforo Mehlich $1,{ }^{5}$ Fósforo Resina, ${ }^{6} \mathrm{KCl} 1 \mathrm{~mol} \mathrm{~L}{ }^{-1}$ e ${ }^{7} \mathrm{SMP}$. SB $=$ Soma de bases trocáveis, $\mathrm{t}=$ Capacidade de troca catiônica efetiva, $\mathrm{CTC}=$ Capacidade de troca catiônica a pH 7,0, e V = Índice de saturação de bases. CV = coeficiente de variação em \% e EPM = Erro padrão da média.

Ciência Rural, v.39, n.7, out, 2009. 
Tabela 3 - Concentração e distribuição de fósforo nos solos das oito lavouras de café Conilon da região Norte do Espírito Santo, cultivados sob sistema orgânico desde o plantio.

\begin{tabular}{|c|c|c|c|c|c|c|c|c|c|c|c|}
\hline \multirow{3}{*}{$\begin{array}{l}\text { Lavoura } \\
1\end{array}$} & \multicolumn{2}{|c|}{----Pi (mg dm³)---- } & \multicolumn{2}{|c|}{----Po (mg dm $\left.{ }^{-3}\right)$---- } & \multicolumn{2}{|c|}{$--\mathrm{Pi}+\mathrm{Po}\left(\mathrm{mg} \mathrm{dm}^{-3}\right)--$} & \multirow{2}{*}{$\begin{array}{c}\text { Rec } \\
\%\end{array}$} & $\mathrm{Pi}$ & \multirow{2}{*}{$\begin{array}{c}\text { Po } \\
\text { otal--- }\end{array}$} & \multirow{2}{*}{$\begin{array}{c}\mathrm{Pi} \\
---\% \text { do }\end{array}$} & \multirow{2}{*}{$\begin{array}{l}\text { Po } \\
\text { ábil--- }\end{array}$} \\
\hline & total & lábil & total & lábil & total & lábil & & ---\% do P total--- & & & \\
\hline & 480,6 & 34,4 & 248,8 & 38,6 & 729,4 & 73,0 & 111 & 65,9 & 34,1 & 47,1 & 52,9 \\
\hline EPM & 3,5 & 0,62 & 3,5 & 8,72 & 3,0 & 8,13 & - & - & - & - & - \\
\hline 2 & 183,8 & 14,1 & 243,1 & 23,1 & 426,9 & 37,2 & 60 & 43,1 & 56,9 & 37,9 & 62,1 \\
\hline EPM & 3,6 & 0,72 & 22,1 & 10,1 & 21,7 & 9,37 & - & - & - & - & - \\
\hline 3 & 579,5 & 46,0 & 310,5 & 21,4 & 890,0 & 67,5 & 96 & 65,1 & 34,9 & 68,1 & 31,9 \\
\hline EPM & 19,4 & 0,52 & 29,5 & 1,67 & 20,8 & 1,20 & - & - & - & - & - \\
\hline 4 & 647,4 & 33,9 & 263,1 & 18,3 & 910,4 & 52,2 & 100 & 71,1 & 28,9 & 64,9 & 35,1 \\
\hline EPM & 39,1 & 0,17 & 132 & 2,17 & 137 & 2,31 & - & - & - & - & - \\
\hline 5 & 212,1 & 8,1 & 259,0 & 17,0 & 471,2 & 25,1 & 54 & 45,0 & 55,0 & 32,3 & 67,7 \\
\hline EPM & 50,0 & 0,19 & 98,0 & 1,44 & 58,9 & 1,59 & - & - & - & - & - \\
\hline 6 & 256,1 & 11,1 & 262,4 & 17,4 & 518,5 & 28,6 & 61 & 49,4 & 50,6 & 38,8 & 61,2 \\
\hline EPM & 4,6 & 0,12 & 13,6 & 1,94 & 13,7 & 2,03 & - & - & - & - & - \\
\hline 7 & 306,8 & 11,3 & 205,2 & 29,9 & 512,0 & 41,3 & 58 & 59,9 & 40,1 & 27,4 & 72,6 \\
\hline EPM & 10,2 & 0,07 & 13,9 & 6,57 & 13,6 & 6,60 & - & - & - & - & - \\
\hline 8 & 299,4 & 10,7 & 199,9 & 12,7 & 499,3 & 23,4 & 51 & 60,0 & 40,0 & 45,7 & 54,3 \\
\hline EPM & 18,6 & 0,03 & 10,5 & 1,74 & 9,2 & 1,77 & - & - & - & - & - \\
\hline $\mathrm{CV}$ & 11,5 & 3,2 & 43,6 & 42,2 & 24,4 & 22,1 & - & - & - & - & - \\
\hline
\end{tabular}

Pi = Fósforo inorgânico, Po = Fósforo orgânico e Rec = recuperação em relação ao P total das amostras. CV = coeficiente de variação em \% e EPM = Erro padrão da média.

bem como a adição de dejeto líquido de suínos ao solo provoca acúmulo de $\mathrm{P}$ nas frações inorgânicas lábeis (GATIBONI et al., 2008). A maior participação do Po lábil, representando $72 \%$ do $P$ total lábil, foi observada na lavoura 7. Porém, na lavoura 3, o Po representou somente $32 \%$ em relação ao $P$ total lábil. A participação do P lábil foi superior em solos submetidos ao cultivo mínimo quando comparado a outros modelos com maior revolvimento (NUÑES et al., 2003). No caso avaliado, por se tratar de cultivos permanentes, o reduzido nível de revolvimento do solo por arados e grades pode estar contribuindo para a grande participação dos componentes orgânicos de P.

O método empregado para a avaliação da distribuição do P do solo (BOWMAN, 1989) possibilitou uma recuperação elevada de $\mathrm{P}$ com quantidades de $\mathrm{Pi}+\mathrm{Po}$ que variaram de 51 até $111 \%$ do P total. Níveis similares de recuperação do método foram observados por GUERRA et al. (1996) para distintos solos do Sudeste brasileiro (48\% a 109\%) e por CUNHA et al. (2003) para mesmo tipo de solo submetido a diferentes coberturas vegetais $(63 \%$ a 93\%).

O conteúdo de carbono total (C) dos solos variou de $12,66 \mathrm{~g} \mathrm{~kg}^{-1}$ na lavoura 2 até $26,69 \mathrm{~g} \mathrm{~kg}^{-1}$ na lavoura 3 (Tabela 4). A humina foi a forma predominante de $\mathrm{C}$ em todos os solos estudados, corroborando os resultados de MACHIORI JÚNIOR \& MELO (2000) e MENDONÇA et al. (2001) ao estudarem amostras de solo de cafezais arábica (C. arabica).
Nas frações alcalino-solúveis da matéria orgânica, observou-se variação representativa entre os solos avaliados (Tabela 4). Para as lavouras 1, 2, 3, 5 e 7, a relação entre o conteúdo de $\mathrm{C}$ nos $\mathrm{AH}$ e $\mathrm{AF}$ apresentou comportamento semelhante ao observado por CANELLAS et al. (2000), pois, de maneira geral, o baixo conteúdo de bases trocáveis nos solos mais intemperizados diminui a intensidade dos processos de humificação, resultando em baixa formação de compostos húmicos. Assim, os resultados permitem inferir que os manejos adotados nas lavouras 1, 2, 3, 5 e 7 favoreceram a formação de substâncias humificadas mais evoluídas, podendo indicar melhoria na qualidade da matéria orgânica do solo, segundo LABRADORMORENO (1996) e CANELLAS et al. (2000).

A relação de humificação variou de 0,07 na lavoura 6 até 0,16 na lavoura 2 (Tabela 4), o que pressupõe que os diferentes manejos e/ou diferentes características dos solos estudados estão influenciando as características do húmus. A diferença encontrada sugere que o material adicionado ao solo possui diferentes graus de polimerização, indicando diferente estabilidade estrutural da matéria orgânica e sua evolução (LABRADOR-MORENO, 1996).

A análise de correlação entre as variáveis indicou uma associação positiva e significativa entre a soma de bases e as diferentes frações da matéria orgânica humificada (Tabela 5). A capacidade de troca 
Tabela 4 - Concentração e distribuição das frações de carbono nos solos das oito lavouras de café Conilon da região Norte do Espírito Santo, cultivados sob sistema orgânico desde o plantio.

\begin{tabular}{|c|c|c|c|c|c|c|c|c|c|}
\hline Lavoura & C & AF & $\mathrm{AH}$ & $\mathrm{H}$ & AF & $\begin{array}{c}\mathrm{AH} \\
\text { lag kg }\end{array}$ & H & $\begin{array}{c}\mathrm{AH} / \mathrm{AF} \\
-\end{array}$ & $\begin{array}{c}(\mathrm{AF}+\mathrm{AH}) / \mathrm{H} \\
-\end{array}$ \\
\hline 1 & 15,88 & 0,80 & 1,11 & 13,96 & 5,04 & 6,99 & 87,90 & 1,39 & 0,14 \\
\hline EPM & 0,10 & 0,02 & 0,02 & 0,55 & 0,11 & 0,14 & 3,98 & 0,05 & 0,01 \\
\hline 2 & 12,66 & 0,88 & 1,01 & 11,70 & 6,95 & 7,98 & 92,41 & 1,15 & 0,16 \\
\hline EPM & 0,26 & 0,02 & 0,09 & 0,45 & 0,21 & 0,67 & 4,53 & 0,11 & 0,00 \\
\hline 3 & 26,69 & 1,28 & 1,43 & 25,96 & 4,79 & 5,36 & 97,25 & 1,12 & 0,10 \\
\hline EPM & 0,33 & 0,07 & 0,04 & 0,51 & 0,22 & 0,22 & 1,82 & 0,09 & 0,00 \\
\hline 4 & 22,37 & 1,33 & 1,07 & 16,68 & 5,95 & 4,78 & 74,56 & 0,80 & 0,14 \\
\hline EPM & 0,27 & 0,14 & 0,02 & 0,96 & 0,63 & 0,05 & 4,11 & 0,08 & 0,02 \\
\hline 5 & 17,25 & 0,65 & 1,16 & 12,50 & 3,76 & 6,72 & 72,46 & 1,78 & 0,14 \\
\hline EPM & 0,86 & 0,02 & 0,06 & 2,34 & 0,30 & 0,07 & 13,1 & 0,16 & 0,03 \\
\hline 6 & 14,76 & 0,51 & 0,29 & 11,07 & 3,46 & 1,96 & 75,00 & 0,57 & 0,07 \\
\hline EPM & 0,26 & 0,08 & 0,04 & 1,19 & 0,48 & 0,33 & 8,94 & 0,16 & 0,01 \\
\hline 7 & 23,79 & 1,18 & 1,25 & 16,20 & 4,96 & 5,25 & 68,10 & 1,06 & 0,15 \\
\hline EPM & 0,10 & 0,04 & 0,09 & 0,81 & 0,19 & 0,39 & 3,20 & 0,07 & 0,01 \\
\hline 8 & 15,53 & 1,07 & 0,27 & 10,80 & 6,89 & 1,74 & 69,55 & 0,25 & 0,12 \\
\hline EPM & 0,51 & 0,04 & 0,00 & 1,20 & 0,09 & 0,06 & 8,70 & 0,01 & 0,02 \\
\hline $\mathrm{CV}$ & 3,8 & 12,0 & 9,8 & 13,4 & 11,1 & 10,9 & 15,6 & 17,4 & 10,3 \\
\hline
\end{tabular}

$\mathrm{C}$ = Carbono total, AF = Ácidos fúlvicos, AH = Ácidos húmicos, H = Huminas, AH/AF = Relação ácidos húmicos com ácidos fúlvicos e $(\mathrm{AF}+\mathrm{AH}) / \mathrm{H}=$ Relação humificação. $\mathrm{CV}=$ coeficiente de variação em \% e EPM = Erro padrão da média.

Tabela 5 - Significância e valor das correlações de Pearson en tre as variáveis encontradas no solo e nas folhas das oito lavouras de café Conilon da região Norte do Espírito Santo cultivados sob sistema orgâni co desde o planti o.

\begin{tabular}{|c|c|c|c|c|c|c|c|c|}
\hline- & $\mathrm{PF}$ & PM & FR & PiL & $\mathrm{PiT}$ & PoT & PoL & $\mathrm{C}$ \\
\hline $\mathrm{PF}$ & 1 & $+0,74 * *$ & $+0,60 *$ & $+0,84 * *$ & $+0,68 * *$ & $+0,38^{\mathrm{ns}}$ & $+0,211^{\mathrm{ns}}$ & $+0,14^{\mathrm{ns}}$ \\
\hline PM & - & 1 & $+0,39^{\mathrm{ns}}$ & $+0,95^{* *}$ & $+0,90 * *$ & $+0,43^{\mathrm{ns}}$ & $+0,09$ ns & $+0,71^{* *}$ \\
\hline FR & - & - & 1 & $+0,41^{\mathrm{ns}}$ & $+0,23^{\mathrm{ns}}$ & $+0,18^{\mathrm{ns}}$ & $+0,11^{\mathrm{ns}}$ & $+0,09^{\mathrm{ns}}$ \\
\hline PiL & - & - & - & 1 & $+0,90 * *$ & $+0,41^{\mathrm{ns}}$ & $+0,33^{\mathrm{ns}}$ & $+0,58^{*}$ \\
\hline PiT & - & - & - & - & 1 & $+0,13^{\mathrm{ns}}$ & $+0,19$ ns & $+0,65^{* *}$ \\
\hline PoT & - & - & - & - & - & 1 & $+0,02$ ns & $+0,07^{\mathrm{ns}}$ \\
\hline PoL & - & - & - & - & - & - & 1 & $+0,09^{\mathrm{ns}}$ \\
\hline- & $\mathrm{H}$ & $\mathrm{AH}$ & $\mathrm{AF}$ & $\mathrm{pH}$ & HA & SB & $\mathrm{T}$ & $\mathrm{Ag}$ \\
\hline $\mathrm{PF}$ & $+0,46^{\#}$ & $+0,14^{\mathrm{ns}}$ & $+0,31^{\mathrm{ns}}$ & $+0,68 * *$ & $-0,64 * *$ & $+0,71^{* *}$ & $-0,29^{\mathrm{ns}}$ & $-0,38^{\mathrm{ns}}$ \\
\hline $\mathrm{PM}$ & $+0,88^{* *}$ & $+0,55^{*}$ & $+0,62^{*}$ & $+0,76^{* *}$ & $-0,54^{*}$ & $+0,98 * *$ & $-0,04{ }^{\text {ns }}$ & $-0,37$ ns \\
\hline FR & $+0,22^{\mathrm{ns}}$ & $+0,38^{\text {пs }}$ & $+0,34^{\mathrm{ns}}$ & $+0,52 *$ & $-0,45^{\#}$ & $+0,37^{\mathrm{ns}}$ & $-0,28$ п & $-0,38$ ns \\
\hline PiL & $+0,80 * *$ & $+0,52^{*}$ & $+0,53^{*}$ & $+0,78^{* *}$ & $-0,56^{*}$ & $+0,95 * *$ & $-0,07^{\text {Is }}$ & $-0,39^{\mathrm{ns}}$ \\
\hline PiT & $+0,71^{* *}$ & $+0,40^{\mathrm{ns}}$ & $+0,66^{* *}$ & $+0,61^{*}$ & $-0,37^{\mathrm{ns}}$ & $+0,94 * *$ & $+0,13^{\mathrm{ns}}$ & $-0,14^{\mathrm{ns}}$ \\
\hline PoT & $+0,37^{\mathrm{ns}}$ & $+0,29^{\mathrm{IS}}$ & $-0,38^{\mathrm{ns}}$ & $+0,61^{*}$ & $-0,77^{* *}$ & $+0,27^{\mathrm{ns}}$ & $-0,69 * *$ & $-0,82 * *$ \\
\hline PoL & $+0,15^{\mathrm{ns}}$ & $+0,50^{\#}$ & $-0,01^{\mathrm{ns}}$ & $+0,20^{\mathrm{ns}}$ & $-0,00^{\mathrm{ns}}$ & $+0,15^{\mathrm{ns}}$ & $+0,08^{\mathrm{ns}}$ & $-0,14^{\mathrm{ns}}$ \\
\hline C & $+0,90 * *$ & $+0,67 * *$ & $+0,78 * *$ & $+0,26^{\mathrm{ns}}$ & $+0,10^{\mathrm{ns}}$ & $+0,77 * *$ & $+0,53^{*}$ & $+0,10^{\mathrm{ns}}$ \\
\hline $\mathrm{H}$ & 1 & $+0,68 * *$ & $+0,66^{* *}$ & $+0,52 *$ & $-0,22^{\mathrm{ns}}$ & $+0,88 * *$ & $+0,26^{\mathrm{ns}}$ & $-0,18^{\mathrm{ns}}$ \\
\hline $\mathrm{AH}$ & - & 1 & $+0,42^{\mathrm{ns}}$ & $+0,60 *$ & $-0,25^{\mathrm{ns}}$ & $+0,54^{*}$ & $+0,03^{\mathrm{ns}}$ & $-0,45^{\#}$ \\
\hline $\mathrm{AF}$ & - & - & 1 & $+0,19^{\mathrm{ns}}$ & $+0,20^{\mathrm{ns}}$ & $+0,72 * *$ & $+0,62 *$ & $+0,33^{\mathrm{ns}}$ \\
\hline $\mathrm{pH}$ & - & - & - & 1 & $-0,88 * *$ & $+0,66^{* *}$ & $-0,58^{*}$ & $-0,84 * *$ \\
\hline HA & - & - & - & - & 1 & $-0,39^{\mathrm{ns}}$ & $+0,86^{* *}$ & $+0,92 * *$ \\
\hline SB & - & - & - & - & - & 1 & $+0,13^{\mathrm{ns}}$ & $-0,21^{\mathrm{ns}}$ \\
\hline $\mathrm{T}$ & - & - & - & - & - & - & 1 & $+0,87 * *$ \\
\hline Ag & - & - & - & - & - & - & - & 1 \\
\hline
\end{tabular}

$\mathrm{PF}=$ Fósforo foliar, PM = Pósforo Mehlich 1, PR = Fósforo Resina, PiL = Fósforo inorgânico lábil, PiT = Fósforo inorgân ico total, PoT = Fósforo orgânico Total, PoL = Fósforo orgânico lábil, $\mathrm{C}=$ Carbono total, $\mathrm{H}=$ huminas, AH = Ácidos húmicos, AF = Ácidos fúlvicos, HA = hid rogênio mais alumínio, SB = Soma de bases trocávei s, CTC = Capacidade de troca catiônica a pH 7,0, Ag = Porcentagem de argila. \#, *, ** $=10$, 5 , e $1 \%$ de probabilidade, respectivamente. $\mathrm{N}=$ a 24 para as correlações.

Ciência Rural, v.39, n.7, out, 2009. 
de cátions foi, significativamente, relacionada com o conteúdo total de C no solo e com a fração AF, que corresponde à fração humificada da matéria orgânica com maior quantidade disponível de $\mathrm{H}^{+}$para troca catiônica. O fato de haver correlação positiva entre $\mathrm{Pi}$ lábil e $\mathrm{P}$ total com a soma de bases, como relatado por ALMEIDA et al. (2003), e entre fósforo foliar e as concentrações de fósforo no solo determinados pelos métodos Mehlich e Resina e com as frações Pi lábil e Pi total (Tabela 5) indicam que essas formas de determinações de $\mathrm{P}$ no solo estão coerentes com a capacidade de extração desse nutriente pela planta.

\section{CONCLUSÕES}

A adição de resíduos vegetais e adubos fosfatados naturais no solo do cafeeiro orgânico proporcionam o aumento de fósforo disponível no solo. Existe relação direta entre fósforo inorgânico versus fósforo orgânico, independentemente da concentração de fósforo total quantificado. A fração humina constituiu a maior parte da matéria orgânica.

As frações que compõem principalmente a distribuição do fósforo no solo apresentaram discrepâncias entre os sistemas de manejos de produção orgânica de café, permitindo agrupar os solos das lavouras em quatro grupos.

\section{REFERÊNCIAS}

ALMEIDA, J.A. et al. Cor de solo, formas do fósforo e adsorção de fosfato em Latossolos desenvolvidos de basalto no extremosul do Brasil. Revista Brasileira de Ciencia do Solo, v.27, p.985-1002, 2003.

ANA - Agencia Nacional de Águas. A bacia do Rio Doce: características da bacia. Capturado em: 16 de abril 2004. Disponível em: http://www.ana.gov.br/cbhriodoce/bacia/clima.

BOWMAN, R.A. A sequential extraction procedure with concentrated sulfuric acid and diluted base for soil organic phosphorus. Soil Science Society of America Journal, v.53, p.326-366, 1989.

BOWMAN, R.A.; COLE, C.V. Transformation of organic phosphorus substrates in soil as evaluated by $\mathrm{NaHCO}_{3}$ extraction. Soil Science, v.125, p.95-101, 1978.

BRASIL. Presidência da República. Casa Civil Subchefia de Assuntos Jurídicos. Lei no 10831 de 23 de dezembro de 2003. Dispõe sobre a agricultura orgânica e dá outras providências. Diário Oficial [da] República Federativa do Brasil, Poder Executivo, Brasília, DF, 24 de dezembro de 2003.
CANELLAS, L.P. et al. Frações da matéria orgânica em seis solos de uma toposequência no Estado do Rio de Janeiro. Pesquisa Agropecuária Brasileira, v.35, p.233-143, 2000.

CRUZ, C.D. Programa genes (Versão Windows): aplicativo computacional em genética e estatística. Viçosa: UFV, 2001. 648p.

CUNHA, T.J.F. et al. Caracterização e natureza do húmus de latossolos amarelos coesos de tabuleiros na região do recôncavo baiano. Magistra, v.15, p.147-154, 2003.

GATIBONI, L.C. et al. Biodisponibilidade de formas de fósforo acumuladas em solo sob sistema plantio direto. Revista Brasileira de Ciência do Solo, v.31, p.691-699, 2007.

GATIBONI, L.C. et al. Formas de fósforo no solo após sucessivas adições de dejeto líquido de suínos em pastagem natural. Revista Brasileira de Ciência do Solo, v.32, p.1753-1761, 2008.

GUERRA, J.G.M. et al. Conteúdo de fósforo orgânico em amostras de solos. Pesquisa Agropecuária Brasileira, v.31, p.291-299, 1996.

LABRADOR-MORENO, J. La materia orgánica e los agrosistemas. Madri: Ministeria Agricultura, 1996. 176p.

MACCALLISTER, D.L.; CHIEN, W.L. Organic carbon quantity and forms as influenced by tillage and cropping sequence. Comunications in Soil Science and Plant Analysis, v.31, p.465-479, 2000.

MACHIORI JÚNIOR, M.; MELO, W.J. Alterações na matéria orgânica e na biomassa microbiana em solo de mata natural submetido a diferentes manejos. Pesquisa Agropecuária Brasileira. v.35, p.1177-1182, 2000.

MALUCHE-BARETTA, C.R.D. et al. Análise multivariada de atributos do solo em sistemas convencional e orgânico de produção de maçãs. Pesquisa Agropecuária Brasileira, v.41, p.1531-1539, 2006.

MATOS, E.S. et al. Formas de fósforo no solo em sistemas de milho exclusivo e consorciado com feijão sob adubação orgânica e mineral. Revista Brasileira de Ciência do Solo, v.30, p.625-632, 2006.

MENDONÇA, E.S. et al. Cultivo do café em sistema agroflorestal: uma opção para recuperação de solos degradados. Revista Árvore, v.25, p.375-383, 2001.

MURPHY, J.; RILEY, J.P. A modified single solution methods for the determination of phosphate in natural waters. Analytica Chimica Acta, v.27, p.31-36, 1962.

NOVAIS, R.F. de; SMYTH, T.J. Fósforo em solo e planta em condições tropicais. Viçosa: UFV-DPS, 1999. 399p.

NUÑES, J.E.V. et al. Conseqüências de diferentes sistemas de preparo do solo sobre distribuição química e perdas de fósforo de um Argissolo. Bragantia, v.62, p.101-109, 2003.

PARTELLI, F.L. et al. Nutritional diagnosis of the organic Conilon coffee trees (Coffea canephora Pierre ex Froehn): 
suffiency range approach for leaves and soil. Coffee Science, v.1, p.43-49, 2006.

PRIMAVESI, A. Agricultura sustentável: manual do produtor rural. São Paulo: Nobel, 1992. 142p.

SWIFT, R.S. Organic matter characterization. In: SPARKS, O.L. Methods of soil analysis Part 3: chemical methods. Madison: Soil Science Society of America, 1996. p.1011-1020.

SILVA, F.C. da (Org). Manual de análises químicas de solos, plantas e fertilizantes. Brasília: EMBRAPA, 1999. 370p.
THEODORO, V.C.A. et al. Alterações químicas em solo submetido a diferentes formas de manejo do cafeeiro. Revista Brasileira de Ciência do Solo, v.27, p.10391047, 2003.

TOKURA, A.M. et al. Formas de fósforo em solo sob plantio direto em razão da profundidade e tempo de cultivo. Pesquisa Agropecuária Brasileira, v.37, p.1467-1476, 2002.

ZECH, W. et al. Factors controlling humification and mineralization of soil organic matter in the tropics. Geoderma, v.79, p.117-161, 1997. 